\title{
Heide Gerstenberger \\ Strukturen jauchzen nicht. Über die Bewegungsform der Französischen Revolution
}

Zusammenfassung: Zahlreiche Untersuchungen über die Französische Revolution beschäftigen sichmit den materiellen Voraussetzungen und nehmen diese als Ursache für die Entstehung eines revolutionären Prozesses. Die Autorin geht der Frage nach, wie aus den sozialstrukturellen Bedingungen revolutionäre Mentalität hervorging und zieht - im Gegensatz zum etablierten marxistischen Interpretationsmodell - das Fazit: diese entstand nicht aufgrund der Verschärfung materieller Widersprüche, sondern im Zuge der Herausbildung einer revolutionären Öffentlichkeit. Es war diese Form von Öffentlichkeit, die zu einer Strukturvoraussetzung des revolutionären Prozesses wurde.

\section{Grobe Skizze des theoretischen Szenariums}

Vor einiger Zeit war, nicht nur in Frankreich, eine Interpretation der Französischen Revolution dominant, die folgendermaßen lautete: Bis zum August 1789 herrschten in Frankreich feudale Verhältnisse: die gesellschaftliche Form der Produktion wurde von grundherrschaftlichen Formen der Aneignung dominiert und die Organisation politischer Gewalt von mittelalterlichen Institutionen. Die Widersprüche dieser Gesellschaft waren unauflöslich, die Revolution stand deshalb seit langem auf der Tagesordnung. 1788 bahnte sich die nationale Krise an. Zur strukturentscheidenden Krise kam es, weil die sich verschärfende soziale Krise mit einer Finanzkrise der politischen Herrschaft zusammenfiel. Letztere war Ausdruck der Tatsache, daß die herrschende Klasse unfähig war, das Régime zu verteidigen. In dieser Situation entwickelte sich, zunächst bei einzelnen, spontan eine revolutionäre Mentalität. Sie konnte sich ausbreiten, weil das Interesse an der Beseitigung der herrschenden Verhältnisse die Angehörigen des dritten Standes - trotzaller vorhandenen Differenzen - einte. Im Verlauf der Revolution wurde um die endgültige Abschaffung des Feudalismus und um die Form der neuen politischen Organisation gerungen. Die Revolution verlief zwar in einzelnen Stadien, bildete nichtsdestoweniger »einen Block «. Sein Inhalt ist im Begriff der bürgerlichen Revolution zusammengefaßt. Die Französische Revolution ist das klassische Modell bürgerlicher Revolutionen. ${ }^{1}$

Gegen diese, von marxistischen Historikern entwickelte, sogenannte sozialstrukturelle Interpretation der Französischen Revolution ist in den letzten beiden Jahrzehnten eingewandt worde, im 18. Jahrhundert könne in Frankreich nicht mehr von »Feu- 
dalismus « die Rede sein, die Aneignung aus Grundherrschäft sei nur noch geringfügig, die Kapitalisierung der Produktionsverhältnisse dagegen bereits weit entwickelt gewesen und durch die Revolution nicht beschleunigt worden. Die Ausweitung des Fernhandels, die Entwicklung der Manufakturproduktion und der Montanindustrie seien keineswegs nur von Nicht-Adligen, sondern im Gegenteil gerade auch von Angehörigen des hohen Adels getragen worden. Die Revolution habe die Besitzstrukturen, das Personal in den staatlichen Verwaltungen sowie insgesamt die Eliten kaum verändert. Thr sozialstruktureller Effekt sei keineswegs als revolutionär zu bezeichnen. Im Unterschied zur sozialstrukturellen Interpretation wird die Revolution aus der Aufeinanderfolge politischer Krisen und Entscheidungen erklärt. Bei der Diskussion von Handlungsmotivationen werden soziale Verhältnisse zwar erwähnt, insgesamt fungiert aber politische Chronologie als Kausalität. Die Revolution wird nicht als »ein Block « gesehen, sondern demgegenüber dargelegt, es sei zunächst zu einer liberalen Revolution gekommen und anschließend dann zu verhängnisvollen Abirrungen von jenem friedlichen Weg der Verwirklichung bürgerlicher Freiheiten, der mit den Ereignissen von 1789 eröffnet worden sei. ${ }^{2}$

Die folgenden Ausführungen - sie behandeln einen einzigen Ausschnitt der Fragen nach den Ursachen der Revolution - kritisieren das etablierte marxistische Interpretationsmodell, sind aber nicht dem Lager seiner Gegner zuzuordnen. ${ }^{3}$

\section{Die Revolutionierung der Gesellschaft: Analytisches Konzept und historisches Ereignis}

In der sozialstrukturellen Interpretation der Französischen Revolution wird das analytische (!) Konzept der bürgerlichen Revolution an das Ereignis der Revolution, an eine bestimmte historische Form also, gebunden. Es wird, mit anderen Worten, unterstellt, die Theorie von den grundlegenden (und deshalb: revolutionären) strukturellen Veränderungen, die notwendig waren, damit kapitalistische Formen der Produktion dominant und vorher personaler Herrschaftsbesitz zur öffentlichen Gewalt des Staates werden konnte, müsse sich daran erweisen, daß diese Veränderungen in unmittelbarem zeitlichen Zusammenhang und deshalb (fast) alle auf einmal durchgesetzt wurden. Weil - das ist offensichtlich und unbestritten - 1789 die bisherige Form der (»politischen«) Herrschaft zur bürgerlichen Staatsgewalt revolutioniert wurde, steht und fällt unter diesen Voraussetzungen die Validität des Konzeptes der bürgerlichen Revolution (und materialistischer Geschichtswissenschaft insgesamt) mit dem Nachweis einer Dominanz feudaler Verhältnisse bis 1789. Eine der Folgen dieser Identifikation einer analytischen Kategorie mit einer spezifischen historischen Form ist die Tatsache, daß das Ereignis selbst nicht als erklärungsbedürftig gilt: Die revolutionäre Mentalität wird als Folge sozialstruktureller Bedingungen und als Ursache des »Dramas « der Revolution eingeführt.

Demgegenüber wird im folgenden erläutert, daß die revolutionären Ereignisse, Vorgänge also, die viele Zeitgenossen als das Ende ihrer Welt und andere als den Beginn 
eines ganz neuen Zeitalters erlebten, nicht aus der Verschärfung materieller Widersprüche resultierten, sondern aus der Entstehung einer revolutionären Öffentlichkeit. Diese war nicht nur strukturelle Voraussetzung der Revolution, sie war auch die Form ihrer Bewegung. Der Kampf um die Bestimmung der neuen Ordnung hatte die Form eines Kampfes um die Vorherrschaft in der Öffentlichkeit. Dieses analytische Konzept, so viel sei zur Erläuterung vorangeschickt, verbleibt im Rahmen materialistischer Strukturanalyse. Es stellt der sozialstrukturellen Interpretation der Revolution theoretisch also nicht Handlungstheorie und methodisch nicht Narration entgegen. Vorgeschlagen wird vielmehr eine Präzisierung materialistischer Analysekonzepte. Und zwar in zweierlei Hinsicht: Öffentlichkeiten, kleinere oder größere, verallgemeinerte und miteinander konfligierende Teilöffentlichkeiten, sind der soziale Ort der Interessenskonstitution. Denn Interessen sind nicht mehr oder minder mechanische Reflexe auf materielle Lebensbedingungen, sondern sie entstehen aus der Wahrnehmung und Beurteilung der eigenen Lebenslage. Die Sicht der eigenen Lage erwächst aus Erfahrung (samt Sozialisation) und aus aktuellem Diskurs. Letzteres bedeutet, daß die verbreitete Rede über Lebensbedingungen, daß Gesten, Lieder, Symbole und auch die - etwa in Bauwerken - materialisierten Formen sozialer Auffassungen die Konkretion von Bedürfnissen und deren (veröffentlichte) Manifestation, die Interessen, mit konstituieren. Deshalb lassen sich die Interessen, die im Herbst des Jahres 1789 vertreten wurden, auch nicht zureichend aus den für 1788 ermittelten »sozialen Voraussetzungen « der Revolution erklären. ${ }^{4}$

Der zweite Vorschlag zur Präzisierung des sozialstrukturellen Analysekonzepts bezieht sich auf die konkreten Merkmale der Öffentlichkeit. So kurzlebig manche von ihnen auch waren, so bildeten sie doch Strukturvoraussetzungen des revolutionären Prozesses. Denn die Formen der Öffentlichkeit - ihr eher literarischer Charakter oder die Manifestation in körperlicher Präsenz -, die materiellen, sozialen, geschlechtlichen und institutionellen Schranken des Zugangs zum öffentlichen Diskurs sind wichtige Voraussetzungen für dessen Inhalte. In der Situation der Revolution, in der sich die Öffentlichkeit an die Stelle der bisherigen Institutionen von Herrschaft (genauer: der Besitzer personaler Herrschaft) setzte, bedeutete die Vorherrschaft über die öffentlichen Diskurse die Entscheidung über den Gang der Revolution. In der Französischen Revolution wurden nicht einfach soziale Widersprüche und Kräfteverhältnisse ausgefochten, es standen sich nicht Klassen und noch nicht einmal Stände gegenüber. Vielmehr ging es um die Durchsetzung von Interessen, die im Zusammenhang öffentlicher Diskurse konstituiert und verändert wurden. Die revolutionäre Öffentlichkeit war die soziale Voraussetzung dafür, daß - an manchen Tagen oder in manchen Stunden - Männer und Frauen »über sich selbst« (und damit auch über ihre soziale Lage) hinauswuchsen. Die Entstehung einer revolutionären Öffentlichkeit hat - selbstverständlich - strukturelle Voraussetzungen: Sie liegen in den materiellen Bedingungen der Reproduktion und in der Praxis von Herrschaft. Aber der revolutionäre Diskurs war kein bloßer Reflex dieser Voraussetzungen. Vielmehr wurden - umgekehrt - die konkreten Bedingungen des Lebens erst im öffentlichen Diskurs zu Gründen für revolutionäre Verhaltensweisen. Einem möglichen Mißverständnis ist vorzu- 
beugen: Im folgenden wird keine »Soziologie der Revolutionen« vorgeschlagen, kein allgemeingültiges Modell für den Ablauf des Dramas von revolutionären Ereignissen. (Ein solches Verlaufsmodell ist die Zielsetzung der einflußreich gewordenen Arbeit von Theda Skocpol [1979].) Eine revolutionäre (Präsenz-)Öffentlichkeit kann zwar als Voraussetzung aller Revolutionen, die in der Form des revolutionären Ereignisses erfolgen, angenommen werden. Aber die Formen und die Inhalte öffentlicher Diskurse gehen immer aus ganz konkreten historischen Situationen hervor. Es mag wohl sein (jedenfalls soll darüber an dieser Stelle nicht gestritten werden), daß bisher alle großen Revolutionen »ihre Kinder gefressen« haben. Eine theoretisch abgesicherte Begründung für die Strukturnotwendigkeit eines derartigen Verlaufs folgt daraus aber nicht.

\section{Von der Bewegungsform des Ancien Régime zur Bewegungsform der Revolution}

Die Bewegungsform aller Gesellschaften vom Typ Ancien Régime war die Praxis personaler Herrschaft ${ }^{5}$ : die Anwendung von Gewaltmitteln (herrschaftlich sanktionierte Privilegien eingeschlossen), die Personen zu eigen waren. Solche Herrschaftspraxis reichte von der Ausübung grundherrschaftlicher Gerichtsbarkeit über die möglichst einträgliche Nutzung von (ererbtem, erworbenem oder delegiertem) Amtseigentum bis hin zur Praxis monarchischer Herrschaft. In der Form der Revolte, der Petition, des verallgemeinerten widerständigen Verhaltens oder auch der-mehr oder minder regelmäßigen - Beratung der Eigner personaler Herrschaft waren Öffentlichkeiten ein Merkmal der Praxis personaler Herrschaft. Solange das Ancien Régime währte, blieben sie dieser Herrschaft jedoch unterworfen. Entsprechendes gilt für die »Öffentlichkeiten «, die den Besitzern personaler Herrschaftsgewalt als Adressaten galten, wenn sie ihre Herrschaft in Symbolen repräsentierten oder auch - seltener bestimmte Entscheidungen begründeten. Verallgemeinernd und verkürzt: Öffentlichkeiten setzten der Herrschaft im Ancien Régime nicht die Inhalte, sondern die Grenzen.

In Frankreich gab es, mehr noch als in den meisten anderen Königreichen und Fürstentümern des Ancien Régime, immense regionale und lokale Unterschiede in den Formen und in der Praxis personaler Herrschaft. Das Ausmaß und die Formen von Grundherrschaft, die Arten und das Maß der finanziellen Belastung durch Steuern samt Abgaben an die Kirche, ständische Institutionen, Gesetze (coutûmes) und Verwaltungsstrukturen differierten von Provinz zu Provinz, von Stadt zu Stadt. Unterschiede gab es vielfach auch zwischen Dörfern und einzelnen Grundherrschaften. Dieser Parzellierung der Herrschaftspraxis entsprach auch diejenige ihrer Öffentlichkeiten. Überlokal verallgemeinert wurden Öffentlichkeiten durch Kriege, durch die (in der Regel nicht im ganzen Königreich gleichförmige) Erhöhung bestehender und die Einführung neuer Steuern und Teuerungen. Seit den Religionskriegen gab es in Frankreich kaum überlokal und überständisch verallgemeinerte religiöse Diskurse. 
Jedenfalls bildeten sie, anders als etwa in England und in den deutschen Fürstentümern, kein Strukturmerkmal der Herrschaftspraxis. Zwar entstanden im Jansenismus (in seinen verschiedenen Entwicklungsstadien unterschiedlich ausgeprägt) Diskurse zur Reform des Glaubens - und der Herrschaftspraxis. Sie blieben aber ständisch und lokal eng begrenzt. ${ }^{6}$

Sowohl von Gegnern als auch von Befürwortern der Französischen Revolution wurde die literarische Öffentlichkeit derAufklärung als eine im ganzen Königreich verallgemeinerte Voraussetzung der Revolution ausgegeben. »Der Absolutismus bedingt die Genese der Aufklärung, die Aufklärung bedingt die Genese der Revolution « schrieb, wie zum Abschluß dieser Tradition, Reinhart Koselleck (1959/1979). Die Aufklärung war-insoweit ist Koselleck zu folgen-ein Strukturmerkmal des Ancien Régime. Ihre Träger setzten der Herrschaft - insbesondere durch die Kritik an Lebensformen des hohen Adels - spezifische Grenzen. Sie drängten aber nicht darauf, eine aufgeklärte Öffentlichkeit zum Subjekt von Herrschaft zu machen. Auch war die literarische Öffentlichkeit in Frankreich, das hat die Begeisterung über die rasche Verbreitung der Enzyklopädie in den Jahren nach 1751 lange verkennen lassen, vergleichsweise begrenzt. Die Entwicklung literarischer Öffentlichkeiten war durch deren warenförmige Strukturen geprägt. Das heißt, daß diejenigen, die über die Mittel zur Herstellung von Druckwerken verfügten, die Möglichkeiten ihrer Profite kalkulierten. In Frankreich wurden im 18. Jahrhundert zwar Veröffentlichungsformen entwickelt, durch welche die Zensurinstanzen umgangen werden konnten, dennoch wurden die Profite überwiegend aus der Ausnutzung herrschaftlich sanktionierter Privilegien gemacht und nicht etwa durch den Versuch, größere Käuferschichten zu erreichen. In einervergleichenden Untersuchung zu dominanten Inhalten und zur Verbreitung von Zeitschriften haben Stephan Botein, Jack R. Censer und Harriet Ritvo (1985) aufgezeigt, daß nicht nur die Anzahl der Zeitschriften in Frankreich vergleichsweise gering war, sondern daß sie sich mit ihren Themen auch nur an eine sozial begrenzte Schicht wandten. Bis 1788 hat sich die literarische Öffentlichkeit in Frankreich in den Grenzen der herrschaftlichen Regulierung von Aneignungsbedingungen entwickelt. Die literarische »politische « Öffentlichkeit war, in dieser These sind die hier nur skizzenhaft möglichen Ausführungen zusammenzufassen, keine Trägergruppe für eine der Reyolution voraufgehende Verallgemeinerung von Herrschaftskritik. Gegenüber allen Ausführungen, in denen die materiellen Strukturen literarischer Öffentlichkeit, der Zustand von Wegen, das Fehlen einer einheitlichen Landessprache und die zumeist eng begrenzten Interessen von Verfechtern und Anhängern der Aufklärung außer Acht gelassen sind, ist schließlich auch daran zu erinnern, daß für viele arme Landbewohner die Revolution mit einem Gerücht beginnen sollte. In der tradierten Form des Hörensagens verbreitete sich - ungeheuer schnell - die Nachricht, Räuberbanden seien im Anmarsch. Es handle sich um ein Komplott der Aristokraten, dem zu begegnen, die Bauern sich bewaffnen müßten. ${ }^{7}$ Die »große Furcht« des Jahres 1789 ist ein deutlicher Ausdruck der Strukturmerkmale von Öffentlichkeiten im Ancien Régime.

Eine weitere Voraussetzung für die Verallgemeinerung von Diskursen über die Praxis 
monarchischer Herrschaft fehlte in Frankreich. Seit 1614 hatte die Krone ohne Beratung durch die Generalstände regiert. Das heißt nicht, daß sie über die - in den verschiedenen Landesteilen unterschiedlichen - Bedingungen ihrer Herrschaftspraxis nicht informiert worden wäre. Es gab Verhandlungen mit Provinzialständen, und es gab die Information durch Intendanten. ${ }^{8}$ Auch Steuerverweigerungen, Revolten und Widerstände gegen Soldatenrekrutierungen »informierten« die Krone über die Bedingungen ihrer Herrschaft. Es gab in Frankreich aber nicht jene - mehr oder minder regelmäßigen - ständisch beschränkten, aber öffentlichen Diskurse über die Formen verallgemeinerter Herrschaft, wie sie durch die Einberufung von Generalständen provoziert werden konnten. Unter dieser Bedingung: Der wenig ausgebildeten Öffentlichkeit zur Herrschaftspraxis der Krone - wurde die bloße Forderung nach einer Einberufung der Generalstände zu einem Akt der Herrschaftsopposition. Nicht in allen Gesellschaften vom Typ Ancien Régime, wie Reinhart Koselleck (1959/1979, S. 49-68) meint, wohl aber in Frankreich, existierte der Diskurs über die Legitimation und die Praxis verallgemeinerter Herrschaft vorwiegend in der Form des Geheimnisses und - so wäre Koselleck zu ergänzen - in derjenigen der Revolte. Es war aber gerade das weitgehende Fehlen einer verallgemeinerten Herrschaftsöffentlichkeit, das den Auseinandersetzungen um die (im Jahr 1787 begonnene) Reform der Provinzialverwaltungen ${ }^{9}$ und dann vor allem auch der Vorbereitung der Generalstände eine Bedeutung verlieh, die zur Entstehung der revolutionären Öffentlichkeit beitrug.

Nun gab es für diese Entwicklung aber noch eine weitere, bereits von Alexis de Tocqueville hervorgehobene Strukturvoraussetzung ${ }^{10}$ : Im Ancien Régime von Frankreich war die Krone zwar lange nicht so durchsetzungsfähig, wie früher von der Geschichtsschreibung angenommen. Die monarchistische Herrschaft war dennoch allgemein. Strukturanalytisch formuliert: Die fortbestehenden partiellen personalen Herrschaftsrechte hatten ihren früheren -faktisch vergleichsweise autonomen-Charakter verloren. Im 18. Jahrhundert war personale Herrschaft - in den ihr verbleibenden Grenzen - der allgemeinen Herrschaft der Krone integriert. Das heißt, sie wurde durch verallgemeinerte Gerichts- und Militärgewalt sanktioniert. (Deshalb war auch Grundherrschaft, soweit sie noch bestand, nicht mehr »private « Feudalgewalt, sondern eine Herrschaftskompetenz, die im Rahmen der verallgemeinerten »staatlichen « Gewalt praktiziert wurde. ${ }^{11}$ ) Die Herrschaftsstrukturen im Ancien Régime von Frankreich waren nicht vereinheitlicht, wohl aber zentralisiert. Und diese Zentralisierung hatte - vor allem in der Form königlicher Fiskalgewalt und in der gesetzlichen Garantie ständischer Privilegien - immerhin derart vereinheitlichende Wirkungen auf die Lebensbedingungen von Menschen, daß die Entstehung eines Diskurses über allgemeine (also nicht nur überindividuell, sondern auch überlokal vorhandene) Interessen historisch möglich wurde. Die Konzeption eines allgemeinen Interesses hat sich historisch (überwiegend) als Kritik an herrschaftlich sanktionierten ständischen Unterschieden entwickelt - in bezug auf die Praxis verallgemeinerter monarchischer Gewalt somit. Die Zentralisierung dieser Herrschaft war Voraussetzung für die Revolutionierung des Herrschafts»systems «, sie war auch die Voraussetzung dafür, daß die 
Öffentlichkeit in Paris, obwohl keinesfalls alleine ausschlaggebend, für die Entwicklung der Revolution von entscheidender Bedeutung werden konnte.

Die organisatorischen Voraussetzungen für die Entstehung einer revolutionären Öffentlichkeit wurden von der Krone geschaffen, als sie die Vorbereitung der Generalstände anordnete. Seit Albert Mathiez' Arbeiten haben sich Historiker angewöhnt, die Versammlung der Notablen im Jahre 1787 als Beginn der Revolution zu betrachten. Diese Versammlung, deren Teilnehmer von der Regierung ausgewählt worden waren, sollte Steuern zustimmen und dadurch deren Eintreibung erleichtern. Indem die Notablen ihre Zustimmung verweigerten und statt dessen die Einberufung der Generalstände zur Beratung über die ihrer Ansicht nach erforderlichen Beschränkungen der königlichen Prärogative forderten, zwangen sie - unter den Bedingungen der Finanzkrise - der Krone eine Politik auf, die zur Herstellung einer verallgemeinerten Herrschaftsöffentlichkeit führte. Die lokalen Versammlungen, in denen, altem Brauch folgend, Kümmernisse ermittelt und in cahiers de doléances niedergelegt werden sollten, waren sozial und geschlechtlich begrenzt. Wer nicht den Rechtsstatus eines Freien hatte, wer in einem Dienstverhältnis stand oder zu den Bettlern und Vagabunden gerechnet wurde, war ebenso wie alle Personen weiblichen Geschlechts von den Beratungen ausgeschlossen. Für Paris galten weitere Beschränkungen. ${ }^{12}$ Soweit sich ermitteln lie $\beta$, hat sich etwa ein Drittel derjenigen erwachsenen Männer, die das Recht dazu hatten, an den Beratungen und an den Wahlen lokaler Vertreter für die regionalen Wahlversammlungen beteiligt. Überwiegend wurden von diesen Versammlungen nur sehr konkrete, Einzelheiten lokaler Herrschaftspraxis betreffende, Forderungen erhoben. An eine regelrechte Umwälzung ihrer Lebensbedingungen wagten zumal die Bauern kaum zu denken, jedenfalls nicht öffentlich. Zwar blieben die Kampagne für eine Verdopplung der Repräsentanz des dritten Standes ${ }^{13}$ sowie die Formulierung und die Verbreitung von Muster-Beschwerdeheften nicht ohne Wirkung. Dennoch ist jenen Historikern zuzustimmen, die darauf beharren, den cahiers de doléances sei keine akut revolutionäre Situation zu entnehmen. ${ }^{14}$

Wie also wurde aus der (in diesem Aufsatz nicht diskutierten!) strukturellen Möglichkeit der Revolution ihre historische Realität? Taylor (1973) und Furet (1980, S. 5257) sind überzeugt, die Erklärung sei im Prozeß der Auswahl und gleichzeitigen Radikalisierung einer politischen Elite zu suchen. In den lokalen und den darauffolgenden regionalen Wahlen habe sich eine politische Führungsschicht gegenseitig selbst aufgeklärt und für die Aufgabe, das Königreich zu liberalisieren, ausgebildet. Tatsächlich ist es kaum denkbar, daß sich eine Ständeversammlung dem - in der traditionell eindrucksvollen Form der séance royale erteilten - königlichen Befehl, die Versammlung aufzulösen, widersetzt hätte, wenn die Kampagne um die Vertretungsstrukturen und die »Revolutionsseminare « (Taylor) der Versammlungen nicht vorausgegangen wären. Wie begrenzt ihre Ziele damals im einzelnen auch noch waren, der Mut jener Delegierten, die sich dem König widersetzten, war eine politische Voraussetzung für die Entstehung der revolutionären Öffentlichkeit. Dieser Mut entstand aus dem Augenblick, war aber auch das Produkt vorausgegangener Diskurse. Diese politische Elite, der die Kritiker späterer Phasen der Revolution so gerne das 
Verdienst zuschreiben, durch die Kraft ihrer Argumente eine friedliche Umwälzung der Verhältnisse bewirkt zu haben, hat die Umwandlung einer Herrschaftsöffentlichkeit der Krone in das Sprachrohr der Nation jedoch beileibe nicht unter sich ausgemacht. Die Beteiligung einer breiten Öffentlichkeit am Prozeß der Politik begann nicht erst mit den sogenannten journées, die gemeinhin als Eintritt des Volkes in die Revolution behandelt werden. ${ }^{15}$

\section{Redekunst und wunde Hacken: Zu den Bedingungen der friedlichen Revolution}

Tag für Tag war bei den Beratungen in Versailles eine große Menge Volks anwesend. Da waren viele, die am Ort (und also zumeist vom Hofe) lebten. Die anderen aber kamen täglich aus Paris, manche in Kutschen, die meisten aber zu Fuß und höchstwahrscheinlich in Schuhen, die für solche politischen Wanderungen nicht eben gut geeignet waren. Fast alle mußten auf Schlaf verzichten, viele auch auf ihren Tagesverdienst. Die Öffentlichkeit der Versammlungen war kein bloßes Publikum (im heutigen passiven Sinne des Wortes), sondern sie war ein Element des sozialen Zusammenhangs, in dem die Delegierten ihre Argumente entwickelten. Das Erlebnis einer präsenten und agierenden »öffentlichen Meinung « ist eine Strukturvoraussetzung der Möglichkeit, bislang unerhörte Gedanken auszusprechen, wenn nicht sogar das erste Mal zu denken. ${ }^{16}$ Das heißt aber, daß auch die Delegierten nicht mehr einfach Träger derjenigen Interessen waren, die sie und ihre Wähler Monate und Wochen vorher als die ihren erkannten. Hätten die Delegierten ganz nüchtern ihre materiellen Interessen abgewogen, der Angriff auf die Grundfesten bisheriger Legalität, in der Sprache der Revolution: »Abschaffung des Feudalismus «geheißen, wäre in der Nacht vom 4. auf den 5. August 1789 wohl kaum beschlossen worden. Der briefliche Anpfiff, den der Delegierte von Clermont-Ferrand kurz nach diesem Beschluß vom Beauftragten des dortigen Municipalausschusses erhielt, weil er an der Abschaffung der Taubenschläge und anderer Einkommensmöglichkeiten seiner Mitbürger mitgewirkt hatte, illustriert diesen Zusammenhang. ${ }^{17}$ Vermutlich hatte Gaultier von Biauzat in dieser aufregenden Nacht die Taubenschläge ganz einfach vergessen. Vielleicht hat er sich ihrer auch erinnert, sie nach der flammenden Rede von Mirabeau aber nicht mehr der Einrede für wert befunden.

François Furet und Denis Richet $(1968$, S. 106; 204) vertreten eine Interpretation der Revolution, in welcher der überwiegend gewaltfreie Charakter der Aktionen des Jahres 1789 auf die von einer aufgeklärten Elite getragenen liberalen Zielsetzungen zurückgeführt wird. Dieses Argument unterschlägt die spezifischen sozialen Voraussetzungen der Gewaltfreiheit. Es war nämlich der Zustand der königlichen Armee derart, daß am 16. Juli 1789 der Kriegsrat dem König davon abriet, die um Versailles und Paris vorsorglich zusammengezogenen Regimenter einzusetzen. Ob die Angehörigen dieser Regimenter im Juli 1789 wirklich gemeutert hätten, ist schwer zu sagen. ${ }^{18}$ Entscheidend ist, daß die soziale Distanz zwischen Offizieren und Soldaten die Ein- 
schätzung ihres Verhaltens unmöglich machte. Diese soziale Binnenstruktur der französischen Armee war zustandegekommen, weil die allermeisten Offiziere ihre Ämter zur Bestätigung ihres Adelsrangs und zur Aufbesserung ihrer Einkünfte erworben hatten und sich kaum je bei ihren Regimentern aufhielten. Nicht nur für die Entwicklungen in Versailles und Paris war der Zustand der Armee von entscheidender Bedeutung. Vielmehr wurden die Bauernrevolten und Brotunruhen, deren es in den Jahren zuvor ebensoviele und teilweise umfangreichere gegeben hatte ${ }^{19}$, erst dadurch zum Bestandteil der »Revolution auf dem Lande «, daß die Regimenter kaum noch zu ihrer Niederschlagung eingesetzt werden konnten. Viele Soldaten desertierten, vereinzelt kam es auch zur Weigerung, auf Revoltierende zu schießen. Nur weil die Krone die faktische Verfügung über ihren militärischen Gewaltapparat im Sommer 1789 weitgehend eingebüßt hatte, konnte der »revolutionäre Stil der Politik « ${ }^{20} \mathrm{im}$ wesentlichen durch Reden zustandegebracht werden. Einzelne Soldaten waren jedoch auch unmittelbar an der Herstellung einer revolutioären Öffentlichkeit beteiligt. So etwa, als sie dem Befehl, die Öffentlichkeit von den Beratungen der Nationalversammlung fernzuhalten, nicht Folge leisteten.

Im Verlauf des Ancien Régime hatten sich die Öffentlichkeiten der Wohlhabenden und Gebildeten in Abgrenzung von Öffentlichkeiten des niederen Volkes konstituiert. Spontane - genauer: zeremoniell nicht regulierte - Präsenzöffentlichkeiten waren dadurch der Tendenz nach zu einem für das niedere Volk charakteristischen Typus von Öffentlichkeit geworden. Im revolutionären Ereignis verschmolzen beide Typen der Öffentlichkeit. Die revolutionäre Öffentlichkeit war sowohl literarisch als auch präsent. Öffentliche Meinung kam in Schriften und Reden, in Liedern und im Lachen und auch im Laufen und im geduldigen Beieinanderstehen zum Ausdruck. Wir müssen uns hier beschränken. Zumindest eine Beschreibung sei dennoch eingefügt. Am 11. August 1789 schrieb ein englischer Reisender, Dr. Rigby, an seine Familie:

»Bald nach unserer Ankunft in Paris fanden wir heraus, daß das Palais Royal, ein großes Viereck, das der Herzog von Orléans in letzter Zeit gebaut hat, der Platz war, wo jede politische Nachricht und Auskunft zu bekommen war; denn hier versammelten sich alle Personen, die in dem großen politischen Drama eine Rolle spielten - hier wurden politische Fragen zuerst diskutiert - und volkstümliche Entschlüsse gefaßt und Vorkehrungen getroffen; hier auch liefen die ersten Mitteilungen von der Nationalversammlung, die ihre Sitzungen in Versailles, 12 Meilen von Paris, abhält, ein, und hier waren die im Druck erschienenen Verhandlungen der Versammlung zuerst zu haben. Schließlich kamen hier auch Zeitungen von einer in der Monarchie bisher unbekannten Art ans Licht; hier wurden die mannigfachen Druckschriften über die verschiedenen politischen Gegenstände, die Tag um Tag von allen Seiten herausgegeben wurden, zuerst gelesen. Auf diesen Platz war unsere Aufmerksamkeit am meisten gerichtet, und um uns in Stand zu setzen, häufig Zeugen dieser Vorgänge zu sein, zogen wir in ein in der Nähe gelegenes Hotel. Wir standen früh am Morgen auf und fanden das Palais Royal zu einer Stunde, wo selbst in London die Straßen leer gewesen wären, gesteckt voll. Die Franzosen haben überall die Gewohnheit, sehr früh aufzustehen. Wir fanden das in Calais und bemerkten es besonders in Lille. Aber ihr frühes Aufstehen in Paris hatte nun noch einen besonderen Grund. Die Verhandlungen der Nationalversammlung und die verschiedenen Vorgänge des vorhergehenden Tages und Abends wurden in der Nacht gedruckt und erschienen bei Tagesanbruch, und so verbreitete sich mit dem Herabfluten des Himmelslichts Kunde der interessantesten Art; und da das Palais Royal das Zentrum der Mitteilung war, wurde es infolgedessen beim ersten Tagesanbruch gesteckt voll. Es war besonders interessant, in diesem Augenblick den Eindruck auf die Menge zu beobachten, den Eifer der Menge zu sehen, sich diese Blätter zu schaffen, und die verschiedenen Diskussionen zu hören, die sofort zwischen den verschiedenen Klassen stattfanden, aus denen sich die 
Menge zusammensetzte, und die in viele bunte Gruppen geteilt waren, von denen jede einen oder zwei Redner hatte. Die Adresse der Nationalversammlung an den König war gerade verlesen worden, als wir den Platz betraten, und ebenso die lebhafte Debatte über die Antwort des Königs, Mirabeau war in beiden Verhandlungen hervorgetreten, besonders in der letzteren; der ganze Platz widerhallte von seinem Preis, und der wärmste Tribut des Beifalls wurde der Versammlung für ihre Festigkeit gespendet (zit. in Landauer 1961, S. 100).

Die revolutionäre Öffentlichkeit wurde zu einem neuen sozialen Milieu. Die Erfahrungen, die Menschen in diesem Milieu machten, wurden zu sozialen Voraussetzungen für die Konstitution von Interessen. Doch ist vor Mißverständnissen zu warnen. An der Herstellung revolutionärer Öffentlichkeiten waren zwar Angehörige aller Schichten des dritten und teilweise auch Angehörige der ersten und des zweiten Standes beteiligt. Aber auch in den Formen revolutionärer Präsenzöffentlichkeit standen, wie Rigby berichtete, die »verschiedenen Klassen « häufig gesondert beieinander. Handelte es sich dabei noch um spontane, den vorläufig unerschütterten Selbstverständlichkeiten des Lebens folgende, Sonderungen, so entwickelten die Bessergestellten unter den Revolutionären andererseits von Anfang an auch ein strategisches Verhältnis zur revolutionären Öffentlichkeit. Die Französische Revolution bestätigt damit nur einmal mehr, daß es im historischen Konstitutionsprozeß bürgerlicher Öffentlichkeiten jene - von Habermas unterstellte ${ }^{21}$ - Phase emanzipatorischer Unschuld nie gegeben hat. Bürgerliche Öffentlichkeit konstituierte sich von Anfang an in Abgrenzung gegen das niedere Volk. Selbst in der revolutionären Praxis ist dieser politische Inhalt bürgerlicher Öffentlichkeit lange vor allem Strukturwandel präsent. Von Anfang an ging es nicht nur um die Beseitigung der alten Ordnung, sondern auch darum, der öffentlich praktizierten Vernunft Grenzen zu setzen. Bereits eine Woche nach dem Sturm auf die Bastille empfahl die Versammlung der Bürger des Distrikts Prémontré Vätern, Kaufleuten und Handwerksmeistern, die »gefährliche Neugierde« derer, die ihrer Autorität unterstellt waren, zu unterbinden (Rose 1983, S. 55). Am 26. Juli wurde eine Verordnung erlassen, daß Arbeiter sowie Tagelöhner ohne festen Wohnsitz und auch diejenigen, »auf deren Arbeit die Gesellschaft nicht verzichten kann «, nicht mehr in die Nationalgarde aufgenommen werden sollten. Zwanzig Pariser Distrikte protestierten - ohne Erfolg. Obwohl die Distrikte diese Regelung unterschiedlich auslegten, veränderte sich insgesamt die soziale Zusammensetzung der Nationalgarde (Reinhard 1971, S. 140). Ihre Regimenter waren geschaffen worden, um die politische Autonomie der revolutionären Gremien gegen mögliche Versuche, die alten Verhältnisse gewaltsam wiederherzustellen, zu verteidigen. Sehr schnell wurden sie aber auch zu einem Instrument eines Teils der revolutionären Öffentlichkeit, welches den Schutz des Eigentums gewährleisten und mögliche Weiterungen der Umwälzung verhindern sollte. Zu diesem Zweck wurde versucht, den Einfluß der Pariser Distrikte auf die Nationalgarden zu beschränken. Nach langen, heftigen Auseinandersetzungen gelang dies im Juni 1790 durch die verwaltungsmäßige Zusammenfassung mehrerer Distrikte zu Sektionen. Ab Dezember 1790 wurden nur noch »Aktivbürger « zur Nationalgarde zugelassen, die ärmeren Schichten also ausgeschlossen. Als - am 17. Juli 1791 - die Nationalgarde auf eine Menge feuerte, die das Ende der Monarchie verlangte, war die Umwandlung des bewaffneten Ausdrucks der 
revolutionären Öffentlichkeit in ein Instrument zur Sicherung der inzwischen etablierten politischen und sozialen Hegemonie über diese Öffentlichkeit vollzogen. Maurice Agulhon (1968/1984) hat vorgeschlagen, die revolutionäre Öffentlichkeit anhand ihrer verschiedenen Gesellungsformen zu analysieren. Der hier unterbreitete Begriff der Bewegungsform ist insofern weiter, als derjenige der sociabilité, als er Denkformen und soziale Praxis (einschließlich der Strategien des Ausschlusses) begrifflich zusammenfaßt und sich auch auf die rechtlichen, administrativen und sonstigen institutionellen Regulierungen bezieht. Zur Bewegungsform der Öffentlichkeit gehören aber auch ihre Symbole und - gelegentlich - ihre Behausungen. Als die Nationalversammlung 1791 in die Tuilerien umzog, diente die architektonische Form dem Ziel, das niedere Volk zum (eher passiven) Publikum der Politik zu machen. Denn die Tribünen waren so weit weg, daß die Redner kaum verstanden und den Debatten nur mit großer Mühe gefolgt werden konnte. Für bessere Herrschaften und Zeitungsleute waren günstigere Plätze vorgesehen. Robespierre wies in seiner Rede vom 10. Mai 1791 (1971, S. 425 f.) darauf hin, daß es durch diese baulichen Maßnahmen gelungen sei, die Öffentlichkeit zwar zuzulassen, sie faktisch aber dennoch auszuschließen. Die Veränderungen des sozialen Milieus, in dem die Nationalversammlung Beschlüsse faßte, hatten materielle Gestalt angenommen.

\section{Der Souverän petitioniert}

Im Ansturm einer erneut revolutionären Öffentlichkeit zerbrach im August und September 1792 die besitzbürgerliche Hegemonie über den politischen Diskurs. Unter den Bedingungen der militärischen Bedrohung durch äußere und innere Feinde ließen sich die Regulierungen der politischen Öffentlichkeit, die eine Beschränkung der Revolution sichern sollten, nicht länger aufrechterhalten. ${ }^{22} \mathrm{Im} J a h r \mathrm{I}$, dessen Beginn der Konvent auf den 22.9.1792, den ersten Tag nach dem Beschluß zur Abschaffung der Monarchie zurïckdatierte, und auch noch in den ersten Dekaden des Jahres II war - wenn auch beileibe nicht unangefochten - eine grundlegend andere Praxis politischen Diskurses dominant. Erster Augenschein könnte vermuten lassen, es sei eine Neuauflage der revolutionären Öffentlichkeit des Jahres 1789. Tatsächlich wurden im August und September 1792 spontane Präsenzöffentlichkeiten zu kollektiven Akteuren. Während es sich 1789 aber noch darum gehandelt hatte, die Öffentlichkeit als Bewegungsform der Gesellschaft und damit als Legitimationsinstanz für Politik zu etablieren, wurden 1792 die politisch dominanten Inhalte des politischen Diskurses und die Formen, in denen sich diese Dominanz reproduzierte, angegriffen. Deshalb wurden die bisherigen Strukturen der Öffentlichkeit auch keineswegs durch bloße Spontaneität, sondern im Gegenteil durch alternative Formen der Organisation des politischen Diskurses ersetzt. Die neue, für eine kurze Zeit dominante, Öffentlichkeitsstrategie läßt sich - der Deutlichkeit halber - auf einen einzigen kurzen Nenner bringen: sie revolutionierte den Charakter von Petitionen.

Im Ancien Régime waren Petitionen Bittgesuche, die sich an die Besitzer personaler Herrschaft oder ihre Vertreter richteten, sie in diesem Akt gleichzeitig - im Prinzip 
zumindest - in ihrer Position bestätigend. Einen derartigen Charakter hatten Petitionen, zumal für Bauern, auch nach 1789. Wenn Dorfbewohner die Nationalversammlung darüber informierten, daß die gesetzlichen Ausführungsbestimmungen der » $\mathrm{Ab}$ schaffung des Feudalismus» nicht zur Verbesserung ihrer Lebensbedingungen taugten, so mußten sie nur allzu schnell erkennen, daß - zusammen mit den Bedingungen ihrer materiellen Reproduktion - auch ihr Ausschluß aus dem politischen Diskurs weitgehend unverändert bestehen bleiben sollte. Erneut sahen sie sich zurückgeworfen auf Petitionen an eine Obrigkeit und auf revoltierendes Verhalten. Erst als im Sommer 1790 und im darauffolgenden Winter (vor allem in Teilen der Bretagne und im Lot) Bauern revoltierten und Burgen in Brand setzten - wobei lokale Abteilungen der Nationalgarde, sei es, daß sie sich ohnehin aus Aufständischen zusammensetzten, sei es, daß sie diese zumindest passiv unterstützten, nicht einzusetzen waren -, entsandte die Nationalversammlung Vertreter, damit sie die Bauern mit Reden besänftigten. Gegen die Ausbeutungsstrategien lokaler Grundherren, bürgerlicher ebenso wie adliger, nutzte das wenig, und die Gesetze, die diesen Strategien (Jones 1988, 4. Kap.) zugute kamen, wurden nicht verändert. Für viele Bauern manifestierte sich die Nationalversammlung als ein Gremium, in dem ihre eigenen Interessen nicht nur nicht vertreten waren, sondern auch kein Gehör fanden. Im Herbst 1792 wurde die Petition aus einem Bittgesuch zu einer Form des sozialen Verkehrs zwischen Staatsbürgern umgewandelt. Sie diente nun dazu, die Institutionen der politischen Öffentlichkeit über die Ansprüche selbstbewußter Bürgerinnen und Bürger zu informieren ${ }^{23}$. Gleichzeitig waren Petitionen das Mittel, in dem die fortbestehenden sozialen und geschlechtlichen Beschränkungen der Partizipation am institutionalisierten politischen Diskurs - zumindest partiell - außer Kraft gesetzt wurden. Es handelte sich, kurz, um die regulierte Form, in welcher der Anspruch, daß jedes Interesse die Chance haben müsse, sich vor dem Richterstuhl der öffentlichen Vernunft legitimieren zu können, zum Ausdruck gebracht wurde.

Das waren nicht demütige Untertanen, die petitionierten, sondern es war der Souverän, der sich in Petitionen mitteilte. Petitionen von Volksgesellschaften und Jakobinerclubs waren die Funktionsformen einer direkten Demokratie. Es gab Massenpetitionen von großen Versammlungen und Petitionen einzelner Arbeiter und Arbeiterinnen, die (von den Verwaltungs- und Regierungsinstitutionen) eine Lösung von Arbeitskonflikten erwarteten. Diese Verwaltungsform des $\gg$ Petitionierens $\ll^{24}$ wirkte ansteckend. So haben sich auch Frauen auf diesem Weg selbstbewußt Gehör verschafft. Obwohl ihnen auch im Jahr II der Revolution nicht mehr zugestanden wurde als der Status eines bürgerlichen Rechtssubjekts und sie vom Status einer Staatsbürgerin rechtlich nicht mehr erhielten als den Namen, verhielt sich manch eine dieser citoyennes, als wäre sie berechtigt, sich frei und gleich am politischen Diskurs zu beteiligen. So wandten sich beispielsweise die Arbeiterinnen der städtischen Spinnmanufaktur an die sociétés populaires der Sektionen Marat und des Chalier, damit diese ihnen gegen die städtischen Beamten beistïnden, die ihren Lohn zu kürzen beabsichtigten. Als »sehr konterrevolutionär « bezeichneten die Arbeiterinnen derartige Pläne (Godineau 1986, S. 99). 
Im Verlauf der Französischen Revolution gab es eine Zeit, in der sich, wenn auch immer nur für einen Teil der Bevölkerung ${ }^{25}$, die Sicht der Welt und des eigenen Lebens durch die Beteiligung an einer kollektiven Praxis der Emanzipation veränderte. Die politische Voraussetzung dieser Erfahrungen war die Realität konterrevolutionärer Bedrohung. In dieser Situtation nämlich wurde die Öffentlichkeit aus einer Legitimationsinstanz für politische Entscheidungen zum Handlungsorgan für die Verteidigung der Revolution. Mehr oder minder spontane, in der Verfassung von 1791 jedenfalls nicht vorgesehene, Bürgervereinigungen übernahmen Funktionen der Regierung und gleichzeitig wurde die Armee aus einem Instrument der Politik in eine Institution zur Bestimmung und Praxis von Politik. Letzteres war, das wird später noch deutlich werden, die machtpolitische Strukturvoraussetzung für die Utopie der direkten Demokratie. Getragen wurde die Praxis einer Bestimmung der Politik durch öffentliche Diskurse von Jakobinern und Sansculotten. Eine Zeitlang sah es so aus, als sollten die politischen Radikalen aus dem oberen mit den Aktivisten aus dem unteren Mittelstand zu einer eimzigen politischen Bewegung verschmelzen. Denn die Montagnards (die Bergpartei) im Konvent stützten sich auf die Volksbewegung; die Mitgliedschaft und die Verfahrensweise in den Jakobinerclubs, in denen sich seit 1789 (damals praktisch noch gemäßigte) Radikale zu politischen Debatten zusammengefunden hatten, wurden demokratisiert; Tausende neuer Clubs und Volksgesellschaften wurden nach dem August 1792 in großen und kleinen Städten und - vor allem im Süden - sogar in Dörfern gegründet. ${ }^{26}$ Manche dieser Vereinigungen hatten nicht mehr als eine Handvoll Mitglieder. Dennoch war die Öffentlichkeit dieser Bürgergesellschaften noch sehr viel größer als selbst die Zahl von (annähernd) 26000 vermuten ließe (Mousnier 1969, S. 121). Denn die Debatten waren öffentlich und Bauern haben das Spektakel der Streitgespräche offensichlich ebenso in den Verlauf einer Fahrt in die nahegelegene Stadt eingeplant wie den Viehhandel und einen Besuch im Wirtshaus (Gervais 1986, S. 428). Die Clubs und Volksgesellschaften dienten Agitatoren zur politischen Propaganda, sie übernahmen aber auch selbst die Verteilung von Schriften und die Organisation von Ansprachen in Dörfern und in der Armee. Sie propagierten und organisierten die Aushebung von Freiwilligen, und sie propagierten und erzwangen die Durchführung der Dechristianisierung.

Im Frühjahr 1793 wurde die Bedeutung, welche die Bürgervereinigungen für die Regierung des Landes gewonnen hatten, dadurch anerkannt, daß beschlossen wurde, ihnen fortan das Bulletin de la Convention kostenlos zukommen zu lassen (Boutrier/ Boutry 1988, S. 379). Darüber hinaus gewährte die Regierung finanzielle Unterstützung für Publikationen, die auch wenig zahlungskräftiges Publikum erreichen sollten. Die Strukturbedeutung der marktförmigen Organisation von literarischen Öffentlichkeiten, die bei formaler Freiheit der Presse die Veröffentlichung bestimmter Inhalte faktisch ebenso erschwert wie die Teilnahme zwar lesefähiger, aber unbemittelter Leser an dieser Öffentlichkeit, wurde in dieser Phase der Revolution reduziert. Auch diese Aufhebung der sozialen Trennung zwischen literarischer Öffentlichkeit und Präsenzöffentlichkeit war eine der Voraussetzungen für die Vorstellung von der Einheit der Volksbewegung. In der nationalen Legende Frankreichs wirkt diese Vorstellung 
bis heute nach: Das für Frankreich spezifische Konzept der Nation erwuchs aus dem Kampf gegen die Konterrevolution. Inhaltlich wurde es zwar bereits in den napoleonischen Kriegen gründlich verändert, aber die Zusammenfassung der Kritik am alten System (wie sie für die Entstehung des Konzeptes der Nation auch in anderen Gesellschaften des Ancien Régime charakteristisch ist) vermischte sich Frankreich mit den Erfahrungen einer möglichen demokratischen Praxis. In der Entwicklung bürgerlicher Gesellschaften erwies sich das Konzept der Nation als eine Illusion der Einheit. Als jedoch am 20. September 1792 die kaum ausgebildeten Freiwilligen und die Soldaten der regulären Armee die Parole »vive la nation « untereinander weitergaben und danach -zeitgenössischen Berichten zufolge - keiner von ihnen vor der Kanonade der preußischen Infanterie zurückwich, da verteidigten sie noch ihre Erfahrung einer möglichen Veränderung der Welt zum Besseren. Sie ist auch in den Namen der Republik mit eingegangen. Als »Marianne« wurde sie, wie Maurice Agulhon (1979, 1. Kap.) ermittelt hat, zuerst von Konterrevolutionären bezeichnet. Denn Marianne, das war ein Name, auf den fast ausschließlich Mädchen der niederen Stände getauft wurden. Entsprechend wurde dieser Name auch in Romanen benutzt, um damit den niederen Stand einer Frauensperson anzuzeigen. Indem Konterrevolutionäre versuchten, die Republik als eine Angelegenheit des Pöbels verächtlich zu machen, decouvrierten sie eigenen sozialen Dünkel. Indem sich die Verteidiger der Republik ihrerseits des Schimpfwortes bedienten, hinterließen sie eine Spur ihrer Begeisterung. Sie rühmten sich dieser »Marianne «, viele mit dem Einsatz ihres Lebens. Im Symbol des Namens Marianne kommt die vorübergehende Harmonie der Sansculotten über die öffentlichen Diskurse zum Ausdruck. Die Radikalisierung mancher Handwerker, Kleinhändler, Manufakturarbeiter und Bauern ist zwar durch die Agitation jakobinischer (Bildungs-)Bürger vorbereitet und gefördert worden. Während sich diese soziale Gruppe gegenüber den Militanten aus den unteren Volksklassen jedoch (soweit sich das ermitteln läßt) das politisch-pädagogische Verhältnis einer Avantgarde zu erhalten suchte, entwickelten die Sansculotten sowohl eigene Formen als auch eigene Strategien des öffentlichen Diskurses.

Jakobiner ebenso wie Sansculotten erstrebten eine möglichst vollständige Politisierung der Gesellschaft. Sie galt ihnen als Voraussetzung für die Verteidigung der Republik. Die Formen der direkten Demokratie, die Propaganda in Reden und Pamphleten waren für beide Trägergruppen charakteristisch. Unterschiede zeigten sich vor allem darin, daß Sansculotten es für möglich und notwendig erachteten, den Prozeß der Politisierung durch eine Praxis der Verhaltensänderung abzukürzen. ${ }^{27}$ Sie versuchten, mit anderen Worten, den Habitus von citoyens und citoyennes $\mathrm{zu}$ verbreiten, auf daß aus dieser Übereinstimmung der Verhaltensmuster die Einheit der Politik erwachse. Wenn die Aktivisten der Sansculotten sich selbst durch ihre Kleidung als Vorkämpfer der Gleichheit kenntlich machten, wenn sie in den Straßen besser Gewandete ebenso wie Priester im Habit verfolgten oder im Winter 1793 durchsetzten, daß von Bäckern nur noch eine einzige Brotsorte, das »Brot der Gleichheit« gebacken wurde, so praktizierten sie eine Diktatur, welche die Gleichheit der Staatsbürger erzwingen sollte. Gleichzeitig erweiterten sie dadurch den (geogra- 
phischen) Raum des politischen Diskurses. Straßen und Plätze waren ebenso wie die Versammlungsräume der Volksgesellschaften, der Sektionen und des Konvents Bühnen der Politik. Die Sansculotten machten die Öffentlichkeit zum Kontrollinstrument der Gesellschaft über sich selbst. Während der kurzen Zeit, in der sie die öffentlichen Diskurse dominierten, war die Öffentlichkeit aber gleichzeitig auch das Subjekt der Politik. Im Verlauf der jakobinischen Diktatur verlor sie diese Position. Der Zeitpunkt dieser Veränderung läßt sich nicht datieren. Denn bis zum Ende der jakobinischen Diktatur blieben militante Sansculotten ihre Träger. Sie setzten ihre (vergleichweise autonome) Praxis der Verhaltenskontrolle fort und akzeptierten die regierungsoffizielle Entlarvung von »Verrätern«. Vor allem aber gelang es dem Revolutionstribunal, zahlreiche Militante davon zu überzeugen, daß - angesichts der Größe der Gefahr - die Formen der direkten Demokratie keine ausreichende Gewähr für einen wirksamen Schutz der Revolution böten. Für diese Aktivisten blieb die Regierung das Instrument ihres politischen Willens. Gegen Ende der Diktatur war diese Überzeugung aber kaum noch in politischer Praxis begründet und insoweit zur politischen »Othodoxie« (R. C. Cobb) geworden. Im Winter 1792/1793 beherrschten militante Sansculotten und Jakobiner die politischen Diskurse in weitgehender Übereinstimmung. Danach wurden die Sansculotten und mit ihnen die Volksgesellschaften mehr und mehr zu Hilfsorganen der Regierung. Die Anzeichen für diese Veränderung sind zahlreich. Hier sind nur exemplarische Hinweise möglich.

Miteinem Gesetz vom 9. September 1793 wurde die »Permanenz der Sektionen« von Paris untersagt. Fortan sollten sie sich nur noch zweimal die Woche versammeln. Gleichzeitig wurde denjenigen, die sich den Verdienstausfall schlecht leisten konnten, ein Sitzungsgeld von 40 sous zugesprochen. Es war ein meisterhafter Streich. Denn die Einführung des Sitzungsgeldes erweckte den Eindruck, es gehe um die Partizipationsmöglichkeiten für ärmere (und vermutet: radikalere) Sansculotten. Tatsächlich war es aber in der Zeit der täglichen Versammlungen sehr häufig einer kleinen Gruppe von Radikalen gelungen, die Diskussion in den Sektionen derart unter ihre Kontrolle zu bringen, daß die Vertreter gemäßigter Auffassungen es vorzogen, fernzubleiben. In den Auseinandersetzungen über Ansprüche auf das Sitzungsgeld kam es jetzt zur Diskriminierung der $» 40$ sous Patrioten «. Die dadurch entstandene Spaltung der Sansculotten verringerte die politische Durchsetzungsfähigkeit der radikalen Basis (vgl. Burstin 1983). Dennoch gründeten sich im Herbst 1793 zahlreiche neue Volksgesellschaften, die sich zum Ziel setzten, durch ihre Debatten und Aktionen, die Aufhebung der »Permanenz« auszugleichen. Viele von diesen lösten sich aber selbst auf - oder sie verzichteten (vorübergehend, wie sie meinten) auf Zusammenkünfte, als nach der Verhaftung der Hébertistes im März 1794 politisch Gemäßigte in öffentlichen Debatten die Regierung zu kritisieren wagten. Die Beschränkung der Meinungs- und Versammlungsfreiheit erschien vielen Militanten unter dieser Bedingung in einem solchen Maße gerechtfertigt, daß sie lieber auch für sich selbst auf diese Freiheiten verzichten wollten, bevor sie dazu beitrügen, den Feinden der Revolution eine Plattform zu verschaffen (Monnier 1987). 
Im Frühjahr 1794 wurde das Schaffott, auf dem in Paris die Guillotine in Betrieb gesetzt wurde, mehrfach verlagert, zuletzt zur Barrière du Trône reversé. Diese Entfernung der Tötungsmaschine aus dem räumlichen Zentrum der hauptstädtischen Öffentlichkeit symbolisiert, daß das Spektakel des Terrors damals den Charakter eines Festes der Revolution eingebüßt hatte. Zwar kamen nach wie vor Zuschauer. Schließlich wurde auf dem langen Weg zum Schaffott ein Drama zur Aufführung gebracht, in dem die Hauptdarsteller die ihnen zugewiesene Rolle immer nur ein einziges Mal spielten. Aber die Hinrichtung von wirklichen oder vermeintlichen Feinden der Revolution war im Sommer 1794 endgültig zu einem Regierungsakt geworden, bürokratisch vorbereitet und manufakturmäßig zum Abschluß gebracht (Arras 1988, S. 121-133).

In jeder Phase der Revolution nahmen die hegemonialen Inhalte des politischen Diskurses im Schauspiel von Festen einprägsame Gestalt an. ${ }^{28}$ Das Fest des höchsten Wesens, das am 20. Prairial des Jahres II (8. Juni 1794) veranstaltet wurde, war Ausdruck der inzwischen vollzogenen Umwandlung des Verhältnisses von politischer Öffentlichkeit und Regierung. Der Wille des Volkes sollte auch weiterhin die Politik bestimmen, aber nicht so, wie er - höchst unvollkommen - von Gliedern desselben geäußert, sondern so, wie er von den Instanzen der Diktatur interpretiert wurde. Die Notwendigkeit zur Begrenzung der Reichweite des vernünftigen Diskurses ist darin angelegt. Dem entsprach die - vor allem von Robespierre betriebene - feierliche Inszenierung einer Ideologie, die ein »religiöses Gefühl « erzeugen sollte, auf daß sich den Menschen die Vorstellung einpräge, es gäbe eine Macht, die über ihnen stehe und die Vorschriften der Moral sanktioniere. ${ }^{29}$

\section{Von den Piken zum livret $^{30}$}

Mit dem Sturz der Robespierristen am 9. Thermidor des Jahres II (27. Juli 1794) wurde der empirische Wille des Volkes programmatisch wieder in seine Rechte eingesetzt. Damit die Manifestationen dieses Willens jedoch nicht von den politischen und sozialen Zielen der Thermodorianer abwichen, galt es, die 1792 verlorengegangene »besitzbürgerliche « Hegemonie über den politischen Diskurs zurückzugewinnen. Mit einer Veränderung der politischen Institutionen war dieses Ziel angesichts der zwischenzeitlichen Politisierung öffentlicher Diskurse - nicht getan. Für den erfolgreichen Sturz der Robespierristen gab es machtpolitische Voraussetzungen: die Veränderung der militärischen Lage und die Auflösung der Revolutionsarmeen. (Letztere waren zur Niederschlagung konterrevolutionärer Aufstände im Innern und zur Durchsetzung der Kriegswirtschaftspolitik gegen widerständige Bauern eingesetzt worden.) Bevor der Sieg der Thermidorianer als gesichert gelten konnte, mußten aber erst noch die sozialen Formen des bislang hegemonialen öffentlichen Diskurses zerstört werden. Teilweise geschah dies unmittelbar und spontan. Als die bisherigen Repressionsstrategien wegfielen, kam es zu wütenden Verhöhnungen von Symbolen der Revolution, wie etwa den Marat-Büsten (Bowman 
1977, S. 171 f.). In diesen Racheakten wurde auch eine Öffentlichkeitsstrategie kritisiert, die darauf gezielt hatte, den gesamten öffentlichen Raum zur Bühne für die Darstellung der Freiheit zu machen. In der exzessiven Frivolität der Bälle, die Aristrokaten und reiche Stadtbürger veranstalteten, feierten diese ihr Überleben. Die Art und Weise dieser Feste machte aber auch öffentlich, daß die Reichen und Vornehmen gewillt waren, fortan über ihre Verhaltensmuster wieder selbst zu bestimmen. Mit ihrer Mode, ihren Gelagen und Spottliedern sprachen sie dem Verhaltensdiktat des Sansculotten Hohn. Die neue »Sprache« des öffentlichen sozialen Verkehrs entstand im Thermidor aus einer Mischung von Erleichterung und Rache. Thre Stabilisierung ging jedoch beileibe nicht einfach von selbst, gewaltfrei und ohne bewußte politische Strategien vor sich.

Damit die politische Öffentlichkeit die Form eines freien Spiels der Meinungen annehmen konnten, mußten die Voraussetzungen und die Formen der sansculottiden Öffentlichkeitspraxis zerstört werden: Den Einrichtungen der direkten Demokratie wurde der Einfluß beschnitten, die politische Ausweitung der literarischen Öffentlichkeit wurde durch die Wiedereinführung der ungehinderten Wirkung des ökonomischen Konkurrenzprinzips zurückgenommen; der Kodex, den Sansculotten für das Verhalten von Bürgerinnen und Bürgern entwickelt hatten, wurde durch die Vorführung von sozialer Differenz im öffentlichen Lebens ersetzt, und der Beteiligung von Präsenzöffentlichkeiten am Prozeß der politischen Meinungsbildung wurde die Legitimation bestritten. Auf die beiden letzteren Aspekte beschränkt sich die folgende Erläuterung. Durch ihre Angriffe auf Personen, deren äußeres Erscheinungsbild und öffentliches Verhalten darauf hindeuteten, daß sie nicht gewillt waren, das Ideal öffentlicher Gleichheit zu verwirklichen, hatten militante Sansculotten eine Definitionshoheit für öffentlich angemessenes Verhalten beansprucht. In beträchtlichem Maße war dieser Anspruch auch realisiert worden. Mit den spontanen Verstößen gegen die Verhaltensgebote, zu denen es nach dem Thermidor überall kam, war diese Definitionshoheit noch nicht gebrochen. Das bewerkstelligte im Winter 1794/95 jedoch die jeunesse dorée (vgl. dazu Gendron (1979). Das waren junge Leute, die von den Sansculotten verächtlich als muscadins (Stutzer) bezeichnet wurden. Im Verlauf der Revolution, vor allem aber in den ersten Monaten nach dem Thermidor, bildeten sie eine regelrechte politische Bewegung. Aktiv war diese vor allem in Paris, aber auch in einigen anderen großen Städten. Zusammengefunden hatten sich die muscadins, als sie ihre übereinstimmenden Ansichten im - zunächst vergleichsweise offenen, später eher verdeckten und trickreichen - Widerstand gegen die Rekrutierungen erkannten. Ihre Opposition gegen bestimmte Ideale der Revolution stellten die Kaufmanns- und Notariatsgehilfen, Journalisten und andere junge Männer, die sich für etwas Besseres hielten, dann auch dadurch zur Schau, daß sie die inkriminierten Kniehosen (culottes), riesige Krawatten, gepuderte Perücken und auch sonst sehr auffallende Kleidung trugen. Sie trafen sich in bestimmten Cafés und auf bestimmten Plätzen, und wenn sie zu mehreren waren, erlaubten sie sich auch Händel mit Sansculotten. In der letzten Zeit der jakobinischen Diktatur allerdings seltener. Nach dem Sturz der Robespierristen machten die muscadins dann jedoch in den Straßen systematisch Jagd auf alle, 
die wie Sansculotten aussahen. Außerdem inszenierten sie Stuhlschlachten in den Sektionssitzungen oder erzwangen, indem sie die Bühne stürmten, Programmänderungen in Theatern. Sie verlangten die Schließung des Jakobiner-Clubs, und als der Konvent darüber debattierte, prügelten sie die anwesenden Sansculotten und ihre Frauen von den Tribünen. Im Winter 1795, als in Paris der Hunger wütete, hinderten die muscadins Frauen daran, eine Petition beim Konvent einzureichen. Mit der täglichen - von ihnen zum Fest erklärten - Jagd auf Militante übersetzten die muscadins die anti-jakobinische Pressekampagne in die Formen des öffentlichen sozialen Verkehrs. Da die Behörden diese Praxis, samt der Aufstellung und dem Exerzieren regelrechter $\gg$ Regimenter «, bewußt duldeten, hatte die jeunesse dorée die Funktion einer Privatarmee der Thermidorianer. Sie konnte Erfolge verbuchen, weil viele Aktivisten der direkten Demokratie Paris verlassen hatten. Ein Großteil war zum Militär eingezogen worden. (Anders als die muscadins entzogen sich die Sansculotten ihrem Militärdienst nicht mit Hilfe der neu eingeführten Möglichkeit eines ärztlichen Attestes.) Andere hatten die Stadt aus Enttäuschung oder auch um möglicher Verfolgung zuvorzukommen, verlassen. Im Germinal des Jahres III (April 1795) endete die behördliche Nachsicht gegen die jeunesse dorée. Ebenso wie den Sansculotten wurde nun auch den muscadins das Tragen von Waffen untersagt.

Im April und im Mai 1795 (Germinal und Prairial) kam es in Paris (im Germinal auch in den Provinzen) zu Volksaufständen. Sie wurden von der Regierung mit Waffengewalt niedergeschlagen (Tønneson 1959/1978, S. XI u. passim). Das war möglich, weil die Soldaten nicht mehr am politischen Diskurs beteiligt waren. Als ihnen mitgeteilt wurde, es handele sich um konterrevolutionäre Aktionen, fehlte ihnen die Information, das zu bezweifeln. Mit der Repression der Volksaufstände des Jahres 1795 wurde die Legitimität der Beteiligung von Präsenzöffentlichkeit am Prozeß der politischen Willensbildung (auf Jahrzehnte hinaus wirksam) bestritten. Massenpetitionen wurden damit zu einem Versuch, den rechtmäßigen Weg der Meinungsbildung zu umgehen. Für die politische Strategie, welche darauf abzielte, den revolutionären Prozeß stillzustellen, war die Kriminalisierung jener Bewegungsform der Öffentlichkeit, in der die Revolution von 1789 ebenso wie ihre Radikalisierung im Jahre 1792 vollzogen worden war, zentral. Die Volksaufstände boten Anlaß, die Aktivisten der Jakobinerdiktatur zu verfolgen. Sansculotten wurden hingerichtet, deportiert und eingekerkert. Viele setzten ihrem Leben selbst ein Ende. Vor allem im Süden kam es im Thermidor zu blutigen, teils offiziell, vor allem aber auch privat geführten Rachefeldzügen gegen die Parteigänger der Diktatur (Pollitzer 1967).

Für die weiteren Veränderungen der Bedingungen und Formen öffentlicher politischer Diskurse sind nur skizzenhafte Hinweise möglich. ${ }^{31}$ Die «Direktorien» (Oktober 1795 bis November 1799) bezogen sich -zunächst-auf eine formal freie, faktisch aber eingeschränkte «öffentliche Meinung». Die Teilhabe am institutionalisierten Prozeß der politischen Meinungsbildung wurde beschränkt. Diese Grenzen wurden im Direktorium gewaltsam verteidigt: Gegen Bauern, die sich gegen die Wiedereinführung der Wehrpflicht erhoben, gegen die Babeuvisten, die von einem Aufstand geträumt hatten. Mit dem 18. Fructidoir des Jahres V (4.9.1797) wandelte sich die Öf- 
fentlichkeitsstrategie der dominanten Fraktionen. Fortan beriefen sie sich - gegen royalistische und neujakobinische Wahlerfolge - auf die Staatsraison, auf eine über die Resultate der politischen Meinungsbildung gesetzte Legitimationsgröße also. Insoweit stimmen Direktorium und Jakobinerdiktatur überein, unterschieden sind sie durch die Inhalte der jeweils selbstherrlich definierten Bezugsgröße. Auch darin trafen sich die Regierungen dieser beiden Phasen der Revolution, daß sie Maßnahmen für notwendig erachteten, die ihnen ihre eigene politische Basis entfremdeten. Bei den Jakobinern galt dies vor allem für die Ausdehnung des Maximums auf die Löhne, beim Direktorium für die rigide Kirchenpolitik, eine Zwangsanleihe bei Reichen und die Stabilisierung der Staatsfinanzen durch einen die Rentner schädigenden Teilbankrott. Die proprietaires sahen sich deshalb nach dem napoleonischen Staatsstreich nicht veranlaßt, die Direktorialverfassung zu verteidigen.

Nach dem 18. Brumaire (9.11.1799) erklärte Napoleon, die Revolution sei jetzt auf ihre Grundsätze zurückgeführt worden und deshalb beendet. Bezogen auf die Öffentlichkeitsstrategien gab es sowohl im Konsulat als auch im Kaiserreich tatsächlich formale (!) Anknüpfungen an die Strukturen liberaler ebenso wie radikaler Öffentlichkeitspraxis. Wirksamer als je zuvor im Verlauf der Revolution setzte staatliche Gewalt jetzt die Grenzen öffentlicher Diskurse: Zensurmaßnahmen, polizeiliche Überwachungen einzelner Personen, Verbannungen, Deportationen und Hinrichtungen unterbanden radikale politische Strömungen. Durch die Begrenzung des Wahlrechts auf die Notablen wurde die Teilnahme am institutionalisierten Prozeß der politischen Meinungsbildung weitgehend beschränkt. Das Prinzip der Repräsentation feierte Triumphe. Allerdings setzte sich Napoleon dann aber auch noch über die Resultate derart zustande gekommener Meinungsbildung hinweg. Wichtige Entscheidungen fielen im Wege des »senatus consultus «, der Information eines Gremiums von ernannten, nicht gewählten »Personen des öffentlichen Lebens«. Dagegen wandten sich die Plebizite mit den für selbstverständlich erachteten sozialen und geschlechtlichen Ausnahmen - an alle. In ihnen aber bestätigte sich der Wandel der Öffentlichkeit von der Bewegungsform einer sich selbst revolutionierenden Gesellschaft zum Akklamationsorgan der Politik, genauer: einer Person.

Auch die Revolution hatte den Personenkult gekannt, die Verehrung von Helden und Märtyrern (Vovelle 1982, 1985, Teil III). In politischen Auseinandersetzungen hatte solcher Personenbezug dennoch vorwiegend symbolischen Charakter behalten. (Am deutlichsten wird dies im Kult um den ermordeten Marat und im späteren Versuch, diesen zusammen mit seinen Symbolen zu zerstören.) Gefordert wurden Brot, Verfassungsregelungen, die militärische Mobilmachung des gesamten Volkes; selbst die Auseinandersetzung um die Hinrichtung des Königs galt mehr der Monarchie, dem Ancien Régime und aktuellen politischen Bedingungen, als dem kleinen dicken Mann, dessen Kopf dann abgeschlagen wurde. Napoleon dagegen wurde als Person akklamiert. Das erste Plebiszit war gefälscht (vgl. Langlois 1972). Nach 1804 verzichtete der Kaiser auf weitere Plebiszite wie auch insgesamt zunehmend auf die Legitimation seiner Regierung vor der »öffentlichen Meinung «. Er stützte sich jetzt auf den militärischen und bürokratischen Gewaltapparat und auf die Tatsache, daß bereits 
seit Beginn des neuen Jahrhunderts die Öffentlichkeit zum Publikum seiner Person und des ihn umgebenden staatlichen Gewaltapparates geworden war. Die Personalisierung bildete jedoch nur einen Teil der diktatorischen Herrschaftsbasis. Denn deren Voraussetzungen lagen in der Vernichtung der revolutionären Volksbewegungen und in der Tatsache, daß für die Bessergestellten die Jahre nach der Jahrhundertwende eine Konsolidisierung ihrer materiellen Situation mit sich brachten, die ihnen den ungestörten Genuß der durch die Revolution nicht angetasteten, bzw. durch sie errungenen, materiellen Gütern erlaubte. Für diese Entwicklung gibt es kein deutlicheres Symbol als die Wiedereinführung des livret, d.h. die polizeiliche Kontrolle des Ortswechsels der Arbeitskräfte und ihrer Einhaltung von Arbeitsverträgen. Faktisch wurden diese Vorschriften vielfach umgangen. Das ändert nichts an der Bedeutung des Sachverhalts, daß nun auch die formale Rechtsgleichheit männlicher Staatsbürger wieder beseitigt wurde. Eine weitere Voraussetzung für die Etablierung einer neuen Hegemonie über den politischen Diskurs war die Entpolitisierung des Militärs: die Beseitigung eines Charakteristikums der revolutionären Öffentlichkeit. ${ }^{32}$ Seit der Zeit des Direktoriums waren die Clubs der Soldaten verboten und politische Agitation in der Armee untersagt worden. Soldaten verloren das Recht zur Mitwirkung bei der Offizierswahl, an der Militärjustiz und an der Verwaltung. Nahegelegt wurde ihnen dagegen ein neues - militärisches - Selbstbewußtsein. Auch sollten sie jetzt stolz darauf sein, die Grande Nation und deren Symbolfigur zu repräsentieren. ${ }^{33}$ Mit dem Nationalismus der napoleonischen Ära wurde an die Stelle der sinnlich erfahrbaren Gemeinschaft revolutionärer Öffentlichkeiten endgültig die »imaginierte Gesellschaft « gesetzt ${ }^{34}$, die vor jeder empirischen Öffentlichkeit und vor allen Interessensdifferenzen und -widersprüchen bereits als aggregathafte Referenzstruktur existiert.

\section{Die öffentliche Existenzform des Staatsbürgers: Ein Nachtrag}

Im Floréal des Jahres XII (2.5.1804) wurde François Léon verhört. Er war auf der Polizeipräfektur von Paris erschienen, um sich ein Arbeitsbuch ausstellen zu lassen. Obwohl er, wie er sagte, zu dem Schluß gekommen war, daß einem nichts anderes übrig bleibe, als sich dieser neuen Vorschrift zu fügen, gelang es ihm nicht, sich aller politischen Bemerkungen zu enthalten. Als man ihn daraufhin festsetzte, fanden sich bei ihm mehrere kleine Zettel mit Beschimpfungen des Kaisers und Kritik an den politischen Zuständen. Im Verhör sagte er aus, er verfertige derartige Zettel selbst und klebe sie an die Hauswände, damit es so aussähe, als teile das Volk seine Meinung. Nach dem Grund befragt, erklärte er: »Ich bin sehr republikanisch. Man sagt mir, der 1. Konsul sei Kaiser. Das paßt mir überhaupt nicht... Man sagt, wir seien frei, aber niemand ist es wirklich. Die Arbeiter will man zwingen, sich livrets geben zu lassen, dabei ist man doch ganz gut ohne sie zurecht gekommen...«. François Léon wurde eingesperrt. Am 23. Juli schrieb seine Schwester ein Gnadengesuch. Über den Bescheid und die Dauer der Strafe ist nichts bekannt (vgl. Sibalis 1982, S. 294 ff.). 
Dieser Sansculotte des Jahres XII der neuen Zeitrechnung steht hier für alle jene, die auch im Kaiserreich noch »sehr republikanisch « gesinnt waren. Diejenigen, die sich heute als die Erben ihrer politischen Praxis betrachten, halten ihnen, bei aller Bewunderung und Verehrung, zumeist Illusionen vor. Befangen in den Denkkategorien ihrer handwerklichen Existenz, hätten sie sich eine Realität staatsbürgerlicher Freiheit erträumt. Der Individualismus ihrer ökonomischen Lebensbedingungen habe sie daran gehindert, den grundsätzlichen Widerspruch zwischen politischer Gleichheit und sozialen Gegensätzen zu erkennen. ${ }^{35}$ In den letzten Jahren wurde der empirische Zirkelschluß, der in diese Argumentation eingegangen ist, aufgedeckt. Die Form der materiellen Reproduktion von Sansculotten war nämlich nicht analysiert, sondern umgekehrt aus ihren politischen Konzeptionen geschlossen worden. Genauerer Betrachtung hält die These vom Individualismus der Reproduktionsbedingungen nicht statt, weil die meisten Pariser Sansculotten in Betrieben beschäftigt waren, in denen mindesten zwölf Gesellen arbeiteten. Arbeitsplatzwechsel waren häufig, und insofern kann sich jenes vertraute Verhältnis zwischen Meistern und Gesellen, das zur Erklärung der politischen Konzeptionen von Sansculotten herhalten mußte, schwerlich eingestellt haben. Dem widersprechen auch die zahlreichen und teilweise heftigen Auseinandersetzungen um Arbeitsbedingungen, die in den Jahren und Monaten vor der Revolution ausgefochten wurden (vgl. Sonnenscher 1985).

Im folgenden soll ein weiterer Einwand formuliert werden. Öffentliche Diskurse, so wurde am Anfang dieses Beitrags ausgeführt, bilden den sozialen Ort für die Konstitution von Interessen. Zu ergänzen wäre, daß die Öffentlichkeit auch derjenige soziale Raum ist, in dem sich materielle Lebenslagen in sozialen Status transformieren. Folgen wir der Analyse Richards Sennetts (1977/1983), so hatte das öffentliche Leben, also der soziale Verkehr an Orten, an denen Bekannte und Unbekannte in mehr oder minder ritualisierten Formen miteinander umgehen, im 18. Jahrhundert noch eine grundsätzlich andere Strukturbedeutung als in späteren Entwicklungsphasen der bürgerlichen Gesellschaft. Erst seit der Industrialisierung begegnen sich Sennett zufolge in der Öffentlichkeit zunehmend Menschen, die das Zentrum ihrer Existenz in der Privatsphäre sehen: »Persönlichkeiten«. Die Übereinstimmung von privater und öffentlicher Existenz wird seither sowohl erwartet als auch (in mannigfacher Weise) hergestellt. Im 18. Jahrhundert dagegen war die Öffentlichkeit noch ein Raum, in dem die öffentlichen Rollen eine eigene - über die private gestellte! - Existenz besaßen. Der Verkehr zwischen den Trägern dieser Rollen war durch soziale Distanz (verstanden als die Ausklammerung ganz privater Merkmale derPerson) gekennzeichnet. Das öffentliche Leben war deshalb sowohl mehr als auch anderes, als das Aufeinandertreffen von privaten Individuen. Sennett beschränkt seine historischen Untersuchungen auf die sozial gehobene großstädtische Öffentlichkeit. Aus Robert Muchembleds (1987) Untersuchungen zur Geschichte der Gesten läßt sich jedoch schließen, daß sich Sennets Thesen sogar auf das öffentliche Leben in Dörfern und damit auch auf andere soziale Schichten erweitern lassen. Wird diese, hier leider nur anzudeutende Strukturbesonderheit des öffentlichen Lebens zur Zeit der Revolution in Betracht gezogen, so entfällt die Notwendigkeit, den Aktivisten der Revolution eine illusionäre 


\section{Sicht des Verhältnisses zwischen politischer Freiheit und materiell gegensätzlichen} Interessen zu unterstellen. Statt dessen ist für möglich zu halten, daß Männer und Frauen aus dem Volk eine öffentliche Existenzweise als Staatsbürgerin und Staatsbürger im Bewußtsein ihrer materiellen Existenzbedingungen erstreben. Womöglich galt ihnen ein öffentlicher sozialer Verkehr, dessen ritualisierte Formen im generellen Gebrauch des »Du«, kulminierte, als ein Ziel, für das sich zu kämpfen lohnte.

\section{Anmerkungen:}

1 Die Ausführungen folgen insbesondere Albert Souboul. Von ihm gibt es sehr differenzierte Analysen zu einzelnen Komplexen. Was das allgemeine Interpretationsmodell angeht, so habe ich nicht übertrieben. Vgl. A. Soboul (1973); derselbe (1977), entsprechend auch: C. Mazauric (1970); für eine systematische Kritik an diesem Interpretationsmodell vgl. G. McLennan (1981), 9. Kap.

2 Vgl. vor allem: F. Furet \& D. Richet (1968); F. Furet (1980); zur Kontinuität ein zusammenfassender Artikel: L. Bergeron (1977).

3 Die Debatte zwischen den zunächst intransigenten «Lagern» hat inzwischen (insbesondere auf Seiten marxistischer Historiker) zu Revisionen und besseren Begründungen geführt. Hier ist keine Darstellung dieser Veränderungen möglich. Deshalb ein Hinweis auf eine über die Thematik hinaus wichtige Arbeit: G. C. Comninel (1987).

4 Es scheint aber, als ob viele der Historiker, die in den letzten Jahrzehnten lokalhistorische Untersuchungen zu den gesellschaftlichen Verhältnissen am Vorabend der Revolution vorgelegt haben, insgeheim unterstellen, wenn erst einmal jede Region und Stadt untersucht worden sei, lasse sich die Frage nach den Ursachen der Revolution zweifelsfrei beantworten. Als Beispiele für die vielfach hervorragenden Einzeluntersuchungen vgl.: P. de Saint Jảkob (1960); P. Léon (1966).

5 Die Formulierung enthält zwei, hier nicht auszuführenden Thesen: Zum ersten, daß Herrschaftspraxis (und nicht etwa ein «Klassenverhältnis») die Strukturbedingungen für entwicklungsbestimmte Auseinandersetzungen prägen, solange Ökonomie und Politik noch nicht als gesonderte Sphären existieren. An die Stelle eines marxistischen Strukturalismus wird dadurch die Analyse der unterschiedlichen Entwicklungsbedingungen in den jeweiligen historischen Epochen gesetzt. Zum zweiten, die These, daß sich die Bedingungen gesellschaftlicher Auseinandersetzungen durch die Verallgemeinerung und Rationalisierung personaler Herrschaft in einem derartigen Ausmaß verändert haben, daß es analytisch nicht nützlich ist, sie weiterhin dem Begriff des Feudalismus zuzuordnen. Ich schlage deshalb vor, Ancien Régime als Strukturbegriff für eine historische Epoche zu benutzen. Zu beidem ausführlich demnächst in: Heide Gerstenberger, Die subjektlose Gewalt. Theorie der Entstehung bürgerlicher Staatsgewalt. Münster Februar 1990. In dieser Arbeit werden auch die hier ausgeklammerten Fragen der Ursachen und strukturellen Resultate der Französischen Revolution diskutiert.

6 Zur jansenistischen Kritik am Ancien Régime vgl. Y. Fauchois (1987), aber auch: B. R. Krieser (1978).

7 Vgl. dazu die klassische Untersuchung von G. Lefebvre (1932), auszugsw. abgedr. in: I. A. Hartig, Hrsg. (1979); unter Berücksichtigung der neueren Forschungsergebnisse: P. M. Jones (1988), 67-80.

8 Die Forschungsergebnisse der letzten beiden Jahrzehnte haben die Vorstellungen vom $\gg$ Französischen Absolutismus « als einer von der Politik der Krone bestimmten Herrschaftsprax is und in diesem Zusammenhang auch das Bild der Intendanten gründlich revidiert. Galten die Intendanten früher als Instrumente der Zentralgewalt, so heute sehr viel eher als Mittler zwischen lokalen und zentralen Politiken, wenn nicht sogar als Vertreter lokaler Interessen. Vgl. zusammenfassend: W. Beik (1985).

9 So argumentiert auch. R. Reichardt, in: E. Hinrichs \& E. Schmitt \& R. Vierhaus, Hrsg. (1978).

10 A. de Tocqueville (1856/1978), vor allem 2. Buch.

11 Ich lasse mich hier auf die lange Debatte zum Feudalismus nicht ein, halte allerdings dafür, daß materialistische Geschichtswissenschaft in diesen Auseinandersetzungen mit unzureichenden Kategorien zu verteidigen versucht wurde. Ein guter Überblick über neuere Forschungen (Heuvel ver- 
sucht, sich dem Streit zwischen den Lagern durch systematische Deskription zu entziehen): G. van Heuvel (1982).

12 Für Paris vgl. M. Reinhard (1971), für die Provinzen vor allem: J. M. Constant (1982).

13 Die »Verdoppelung« bezieht sich auf die Repräsentation von 1614, die zunächst auch für 1789 vorgesehen war.

14 Zu den nicht-revolutionären Inhalten der cahiers vgl. G. V. Taylor (1973). Taylor mißt den revolutionären Charakter an aufklärerischen Inhalten, macht dadurch also die Aufklärung zur Ursache der Revolution. Seine Darstellung ist dennoch nützlich. Den Eindruck einer vorrevolutionären Situation erweckt die Auswahl von Forderungen, die P. Goubert \& Denis, M., 1964) getroffen haben.

15 Vgl. hierzu und zum Folgenden: J. Michaud (1960).

16 Die politischen Inhalte ergeben sich nicht aus der sozialen Form der Präsenzöffentlichkeit, sondem aus den Ursachen, die zu derart »bewußtseinserweiternden« Ereignissen führten.

17 Anton Bergier a Gaultier de Biauzat, 13.8.1789, abgedr. in: G. Landauer (1961) 48.

18 Vgl. hierzu die hervorragende Untersuchung von: S. F. Scott (1978); für die sozialen Strukturen der Armee am Ende des Ancien Régime vgl. auch: D. D. Bien (1974).

19 So vor allem der guerre de la farine im Jahre 1795.

20 Ich übernehme diesen Ausdruck von J. G. A. Pocock (1970, S. 280). Pocock macht die nützliche Unterscheidung zwischen einer Rede, die Mittel sozialer Praxis ist, und einer Rede, die als solche ein politisches Ereignis darstellt. Auch seiner Ansicht nach tritt letzteres insbesondere im Zusammenhang von Revolutionen auf.

21 Habermas (1962/1980) diskutiert die Konstitution bürgerlicher Öffentlichkeit als die Politisierung einer bereits frühbürgerlichen literarischen Öffentlichkeit. Sie richtete sich seiner Ansicht nach gegen das Ancien Régime. Die Ausschlußpraktiken gegenüber Präsenzöffentlichkeiten (sowie insgesamt gegen die niedrigeren Stände), die ein Merkmal der historischen Konstitution bürgerlicher Öffentlichkeit bilden, diskutierte Habermas nicht.

22 Ein erster Überblick über den Ablauf ist leicht zugänglich bei: M. Vovelle (1982/1985): Ich muß mich hier mit Literaturhinweisen sehr beschränken, für einen Überblick über neuere deutschsprachige Literatur vgl. R. Reichardt (1989).

23 Auch Bauern, die sich (nachdem sie die Revolution zunächst begrüßt hatten) aufgrund der kriegswirtschaftlichen Abgabeforderungen der Konterrevolution zuwandten oder ihr von den Aktivisten zugeschlagen wurden, richteten ihre Appelle an die »Brüder und Freunde « und hofften auf die öffentliche Vernunft. Belege hierzu bei: Y.-M. Bercé (1974) 146, 184. Zur Bekämpfung des Bauernwiderstandes (vor allem auch durch den Einsatz der Revolutionsarmeen) vgl. R. C. Cobb (1970).

24 Ich übernehme diese Formulierung von M. Godineau (1986, S. 107).

25 Über die zahlenmäßige Beschränkung aktiver Beteiligung vgl. M. Vovelle, op. cit. Teil III, 2.

26 Zum Stand der Forschung über die Verbreitung der Clubs und Volksgesellschaften vgl. das Sonderheft der Annales historiques de la Révolution Francaise, Bd. 58 Nr. 266 (1986), darin vor allem den Beitrag von J. Boutrier \& Ph. Boutry, 364-398.

27 Grundlegend noch immer: A. Soboul (1958), teilw. übers. (1978) In der Beurteilung der sozialen Basis von Soboul abweichend: R. B. Rose (1983); von Sansculottenbewegungen im Plural spricht: R. C. Cobb; in: J. Kaplow (1965) $305 \mathrm{ff}$.

$28 \mathrm{Vgl}$. die inzwischen klassische Arbeit von M. Ozouf (1976); unterschiedliche Interpretationsmodelle in: Les Fétes de la Révolution (1977).

29 Ich paraphrasiere M. Robespierre, op. cit. 667-675.

30 Piken waren häufige Waffen (und Symbole bei Volkserhebungen. Im Laufe der Revolution kamen Sansculottes durch ihre Mitgliedschaft in den Nationalgarden aber auch zu regulärer Bewaffnung. Die Abschaffung der Arbeitsbücher (livrets) war eine der erfolgreich durchgesetzten revolutionären Forderungen. Zur Wiedereinführung vgl. insbesondere: M. D. Sibalis (1979) 6. Kap.

31 Eine Zusammenfassung des Forschungsstandes zum Direktorium: M. Lyons (1975).

32 Zum Strukturwandel der Armee: L. Bergeron (1972, S. 114 ff.)

33 Zur Entwicklung des Konzeptes der Nation: J. Godechot (1966).

34 Die Bezeichnung folgt B. Anderson (1983/1988).

35 So insbesondere bei A. Soboul (1958/1978), weitere Vertreter dieser Interpretation sind aufgeführt bei M. Sonnenscher (1985), Anm. 2). 


\section{Literatur}

Agulhon, Maurice (1984): Pénitents et franc-maçons de l'ancienne Provence. Essai sur la sociabilité méridionale (1968), Paris

Agulhon, Maurice (1979): Marianne au Combat, Paris

Anderson, Benedict (1988): Die Erfindung der Nation, Frankfurt/M. (Imagined communities, London 1983)

Arras, Daniel (1988): Die Guillotine. Die Macht der Maschine und das Schauspiel der Gerechtigkeit (Originalaus. 1987) Reinbek bei Hamburg

Beik, William (1985): Absolutism and society in Seventeenth-Century, France, Cambridge

Bercé, Yves-Maric (1974): Croquants et $N u$-Pieds. Les Soulèvements paysans en France du XVI $I^{c}$ auXIX ${ }^{c}$ siècle, Paris

Bergeron, Louis (1972): L'Episode napoléonien. Aspects intérieurs. Paris

Bergeron, Louis (1977): Die französische Gesellschaft von 1750 bis 1820, in: Zeitschr. für Hist. Forschung Nr. 4 131-146

Bien, D.D. (1974): La réaction aristocratique avant 1789: l'exemple de l'armée, in: Annales E.S.C. Bd. $29,23-48,505-534$

Botein, Stephen \& Censer, Jack R. \& Ritvo, Harriet (1985): La presse périodique et la société anglaise et française au XVIII ${ }^{\mathrm{e}}$ siècle: une approche comparative, in: Rev. d'hist. mod et contemporaine, Vol. 32, 209-236

Boutrier, Jean \& Boutry, Philippe (1988): La diffusion des sociétés politiques en Crance (1789- an III), in: Annales Historiques de la Révolution Française, Bd. 58, Nr. 266, 365-398

Bowman, Franck Paul (1977): Le >Sacré Cœur< de Marat; in: Fêtes de la Révolution, 155-179

Burstin, Haim (1983): Les citoyens des quarante sous. Analyse sociopolitique à l'intérieure de la sansculotterie; in: Annales Hist. de la Rév. Franç. Bd. 55, 93-112

Cobb, Richard C. (1961-1963): Les Armées Révolutionnaires, 2 Bde, Paris - La Haye

Cobb, Richard C. (1965): Some aspects fo the revolutionary mentality April 1793-Thermidor year II; in: Jeffry Kaplow, Hrsg. 305 ff.

Cobb, Richard C. (1970): The Police and the People. French Popular Protest 1789-1820. Oxford

Cobban, Alfred (1964): The social interpretation of the Franch Revolution. Cambridge

Cobban, Alfred (1973): The Myth of the French Revolution, teilw. in: E. Schmitt, Hrsg., 57-72

Comninel, George C. (1987): Rethinking the French Revolution. Marxism and the Revisionist Challenge, London - New York

Constant, J.-M. (1982): Les idées politiques paysannes. Étude comparée des cahiers de doléances (15761789); in: Annales E.S.C. Bd. 37.2, 717-729

Fauchois, Yann (1987): Jansénisme et Politique au XVIII ${ }^{\mathrm{e}}$ Siècle: Légitimation de l'état et légitimation de la Monarchie chez G.N. Maultrot; in: Revue d' hist. moderne et contemporaine Bd, 34, 473-491

Furet, François \& Richet, Denis (1968): Die Französische Revolution, München

Furet, François (1980): 1789 - Vom Ereignis zum Gegenstand der Geschichtswissenschaft, Frankfurt/ Main.

Gendron, François (1979): La Jeunesse Dorée. Quebec

Gervais, P. (1986): L'Autre Bretagne. Les clubs révolutionnaires bretons 1789-1795; in: Annales Historiques de la Révolution Française Bd. 58, Nr. 266, 422-447

Godechot, Jacques (1966): La Grande Nation, 2 Bde, Paris.

Godechot, Jacques (1971): Nation, patrie, nationalisme et patriotisme en France au XVIII siècle; in: Annales Hist. de la Révolution Française, Bd. 43, 482-501

Godineau, Dominique (1986): Travail et Politique à Paris pendant la Révolution: L'Exemple des Ateliers Municipaux; in: Annales Historiques de la Révolution Française, Bd. 58, Nr. 263, 97-111

Goubert, Pierre \& Denis, Michel (1964): 1789. Les François ont la Parole Cahiers des États Généraux, Paris

Habermas, Jürgen (1980): Strukturwandel der Öffentlichkeit, (1962) Darmstadt und Neuwied

Hartig, Irmgard A. (Hrsg.) (1979): Geburt der bürgerlichen Gesellschaft, Frankfurt/Main

Heuvel, Gerd van den (1982): Grundprobleme der französischen Bauernschaft, 1730-1794. Soziale Differenzierung und sozio-ökonomischer Wandel vom Ancien Régime zur Revolution. München - Wien 
Hinrichs, Ernst \& Schmitt, Eberhard \& Vierhaus, Rudolf (Hrsg.) (1978): Von Ancien Régime zur Französischen Revolution, Göttingen

Jones, P.M. (1988): The Peasantry in the French Revolution. Cambridge

Kaplow, Jeffry (Hrsg.) (1965): New Perspectives on the French Revolution. New York

Koselleck, Reinhart (1969): Kritik und Krise (1950), München

Kreiser, B. Robert (1978): Miracles, Convulsion and Ecclesiastical Politics in Early Eighteenth Century Paris, Princeton

Landauer, Gustav (1961): Die Französische Revolution in Briefen. Hamburg

Langlois, Claude (1972): Le Plébiscite de l'an VIII ou le coup d'état du 18 pluviôse an VIII; in: Annales historiques de la Révolution Française, Nr. 207: 43-65; Nr. 208: 231-246; Nr. 209: 390-415

Lefebvre, Georges (1979): Die Große Furcht von 1789 (Auszug aus: La Grande Peur de 1789, 1932), in: I.A. Hartig, Hrsg. 88-135

Les Fêtes de la Révolution (1977): Colloque de Clermont-Ferrand (1974), hrsg. von Jean Ehrard \& Paul Viallaneix, Paris.

Lyons, Martyn (1975): France under the directory, Cambridge

Mathiez, Albert (1950): Die Französische Revolution, 3 Bde, Hamburg (Paris 1922-1927)

Mazauric, Claude (1970): Sur la Révolution Française, Paris

Mc Lennan, Gregor (1981): Marxism and Methodology of History, London

Michaud, Jean (1960): Les États Généraux et le 14 Juillet 1789, Paris

Monnier, Raymonde (1987): La Dissolution des Sociétés Populaires Parisiennes au Printemps de l'An II; in: Annales Historiques de la Révolution Française Bd. 59, Nr. 268, 176-191

Mousnier, Roland (1969): Les Hiérarchies sociales de I450 à nos jours. Paris

Muchembled, Robert (1987): Pour une Histoire des Gestes (XVe - XVIII' Siècle); in: revue d' hist. mod. et contemporaine Bd. 34, 87-101

Ozouf, Mona (1976): La fête révolutionnaire, 1789-1799, Paris

Pocock, John Grenville Angus (1972): Politics, Language and Time. Essays on Political Thought and History (1960), London

Pollitzer, Marcel (1967): Sous la Terreur Blanche. Paris

Reichardt, Rolf (1978): Die revolutionäre Wirkung der Reform der Provinzialverwaltungen in Frankreich 1787-1791; in: E. Hinrichs \& E. Schmitt \& R. Vierhaus, Hrsg., 66-124

Reichardt, Rolf (1989): Von der politisch-ideengeschichtlichen zur sozio-kulturellen Deutung der Französischen Revolution. Deutschsprachiges Schrifttum 1946-1988; in: Geschichte und Gesellschaft, 15. Jg., H. 1, 115-143

Léon, P. (1966): Structures économiques et problèmes sociaux du monde rural dans la France du SudEst. Paris

Reinhard, Marcel (1971): Nouvelle Histoire de Paris. La Révolution 1789-1799. Paris

Robespierre, Maximilien (1971): Ausgewählte Texte. Hamburg

Rose, R.B: (1983): The Making of the Sans-Culottes. Democratic ideas and institutions in Paris I789-92. Manchester

Saint Jacob, Paul de (1960): Les Paysans de Bourgorgne du Nord au dernier siècle de l'Ancien Régime. Paris

Scott, Samuel F. (1978): The Response of the Royal Army to the French Revolution. The Role and Development of the Line Army 1787-93, Oxford

Sennett, Richard (1983): Verfall und Ende des öffentlichen Lebens . Frankfurt/Main

Schmitt, Eberhard (Hrsg.) (1973): Die Französische Revolution. Darmstadt

Sibalis, Michael David (1979): The Workers of Napoleonic Paris. Diss. Paris

Sibalis, Michael David (1982): Un Sans-Culotte Parisien en l'An XII: François Léon, Frère de Pauline Léon, Edition eines Dokumentes; in: Annales Historiques de la Rév. Française Bd. 54, Nr. 247

Skocpol, Theda (1979): States and Revolutions, Cambridge

Soboul, Albert (1973): Die Große Französische Revolution, 2 Bde, Frankfurt/Main (Originalausgabe 1962)

Soboul, Albert (1978): hrsg. von W. Markov, Französische Revolution und Volksbewegung: die Sansculotten, Frankfurt/Main (gekürzte Ausg. von b: Les Sans-culottes parisiens en l'an II, 1958)

Soboul, Albert (1977): Kurze Geschichte der Französischen Revolution, Berlin (Originalausgabe 1965) 
Sonnenscher, Michael (1985): Les sans-culottes de l'an II: répenser le langage du travail dans la France révolutionnaire; in: Annales E.S.C. 1087-1108

Taylor, George V. (1973): Non-Capitalist Wealth and the Origins of the French Revolution (1967), abgedruckt in: Eberhard Schmitt, Hrsg., 288-328

Taylor, George V. (1973): Les cahiers de 1789: aspects révolutionnaires et non révolutionnaires; in: Annales E.S.C., Bd. 28.2, 1495-1514

Tocqueville, Alexis de (1978): Der alte Staat und die Revolution (Originalausgabe 1856) München Tø̆neson, Käre D (1978): La Défaite des Sans-Culottes (1959) Oslo

Vovelle, Michel (1985): Die Französische Revolution - Soziale Bewegung und Umbruch der Mentalitäten (München - Wien 1982) Frankfurt/Main

sanchible $2 A$

Dle Lage nach der Umschichiung durch Entwicklungen der 60 -ar Jahre

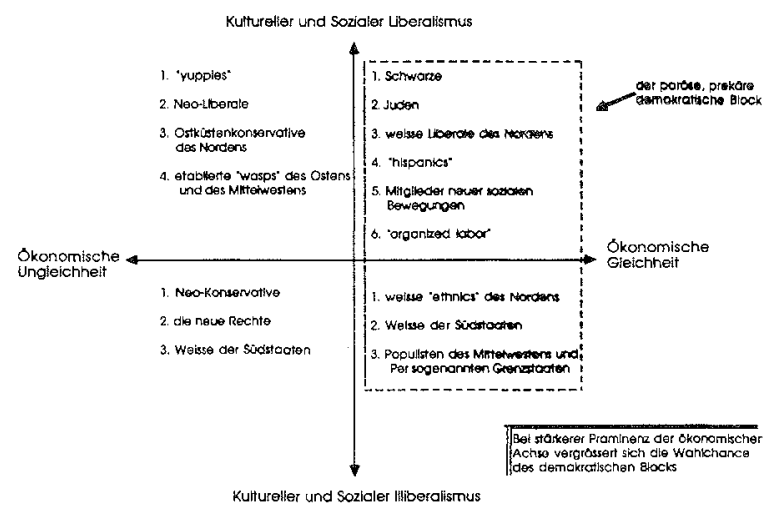

Schaubild $2 B$

Die Lage nach der Umschichtung durch Entwicklungen der 60-er Jahre

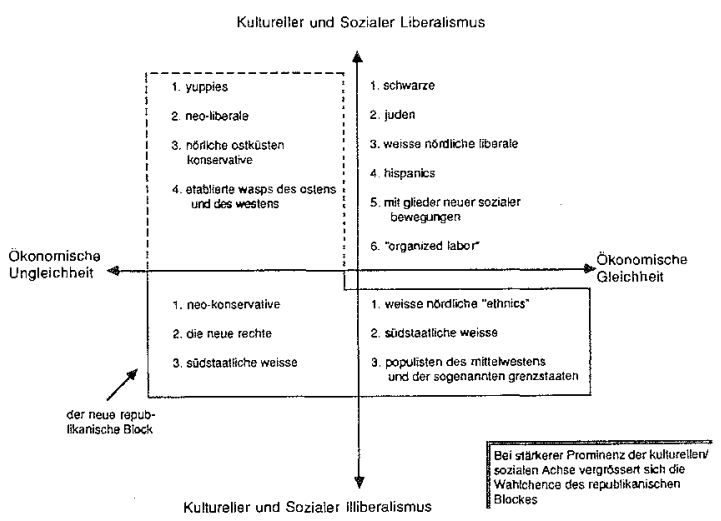

Errata: Leider wurde in der PROKLA $74 »$ Bye, Bye USA in dem Artikel von Andrei S. Markovits: Die Präsidentschaftswahlen 1988, ein sehr wichtiges Schaubild vergessen. Das Schaubild sei hiermit nachgetragen. Die Redaktion bittet für dieses Versäumnis herzlich um Entschuldigung. 